\title{
Dissipative Heating in a Rotational Viscometer with Coaxial Cylinders
}

F. Rieger

Rotational viscometers with coaxial cylinders are often used for measuring rheological behaviour. If the inner to outer cylinder diameter ratio does not differ significantly from 1, the curvature can be neglected and the flow reduces to the flow between moving and stationary plates. The power-law and Bingham models are often used for describing rheological behaviour. This paper deals with the temperature distribution obtained by solving the Fourier-Kirchhoff equation and in the case of negligible inner heat resistance it also covers temperature time dependence. The solution is illustrated by a numerical example.

Keywords: viscous heating, viscometer with coaxial cylinders, power-law fluids, Bingham plastics.

\section{Introduction}

This paper presents viscous heating in a rotational viscometer with coaxial cylinders (see Fig. 1), which is often used for measuring rheological behaviour.

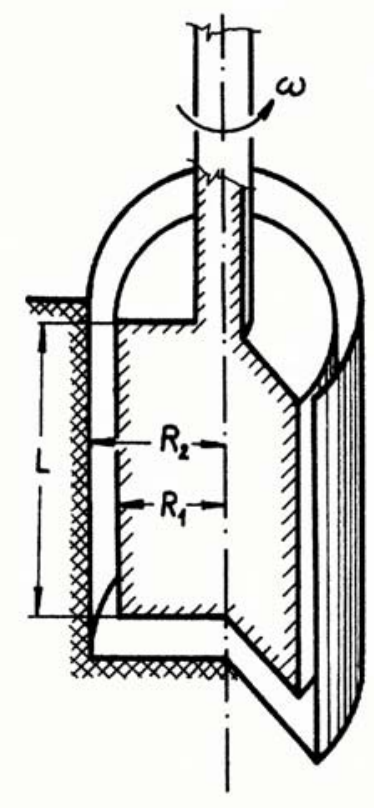

Fig. 1: Rotational viscometer with coaxial cylinders

Power-law and Bingham models are often used to describe rheological behaviour [1].

The power-law model is the simplest model widely used for describing the rheological behaviour of non-Newtonian fluids. Using this model, the dependence of shear stress $\tau$ on shear rate can be expressed by the relation:

$$
\tau=K \dot{\gamma}^{n},
$$

where $K$ is the coefficient of consistency and $n$ stands for the flow behaviour index.

The Bingham model is the simplest model used for describing the rheological behaviour of viscoplastic materials. Using this model, the relation of shear stress $\tau$ and shear rate $\dot{\gamma}$ can be expressed by the following relation:

$$
\tau=\mu_{\mathrm{p}} \dot{\gamma}+\tau_{0} \text { for }|\tau| \geq \tau_{0},
$$

where $\tau_{0}$ is the yield stress and $\mu_{\mathrm{p}}$ stands for plastic viscosity.
If the inner to outer cylinder diameter ratio does not differ significantly from 1 , the curvature can be neglected and the flow reduces to the flow between the moving and stationary plates.

The temperature distribution can be obtained by solving the Fourier-Kirchhoff equation [1]

$$
\lambda \frac{\mathrm{d}^{2} T}{\mathrm{~d} y^{2}}=-\tau \dot{\gamma},
$$

where $y$ is the distance from the stationary plate. The equation will be solved with the following boundary conditions

$$
\begin{aligned}
& y=H, \quad \frac{\mathrm{d} T}{\mathrm{~d} y}=0 \\
& y=0, \quad \lambda \frac{\mathrm{d} T}{\mathrm{~d} y}=\alpha\left(T-T_{\mathrm{f}}\right)
\end{aligned}
$$

i.e., we assume an insulated moving plate (rotating cylinder) and a stationary plate (cylinder) tempered to temperature $T_{\mathrm{f}}$.

\section{Solution}

\subsection{Power-law fluids}

Inserting (1) for $\tau$ into (3), we get

$$
\lambda \frac{\mathrm{d}^{2} T}{\mathrm{~d} y^{2}}=-K \dot{\gamma}^{n+1}
$$

and after integration we obtain

$$
T-T_{\mathrm{f}}=\frac{K H^{2} \dot{\gamma}^{n+1}}{\lambda}\left(\frac{y}{H}-\frac{1}{2}\left(\frac{y}{H}\right)^{2}+\frac{1}{B i}\right),
$$

where $B i=\alpha H / \lambda$. The solution is shown in graphical form in Fig. 2, where

$$
T^{*}=\frac{\left(T-T_{\mathrm{f}}\right) \lambda}{K H^{2} \dot{\gamma}^{n+1}}, \quad y^{*}=\frac{y}{H} .
$$

\subsection{Bingham plastics}

Inserting (2) for $\tau$ into (3), we get

$$
\lambda \frac{\mathrm{d}^{2} T}{\mathrm{~d} y^{2}}=-\frac{1}{\lambda}\left(\mu_{p} \dot{\gamma}^{2}+\tau_{0} \dot{\gamma}\right)
$$

after integration and rearrangement we obtain 


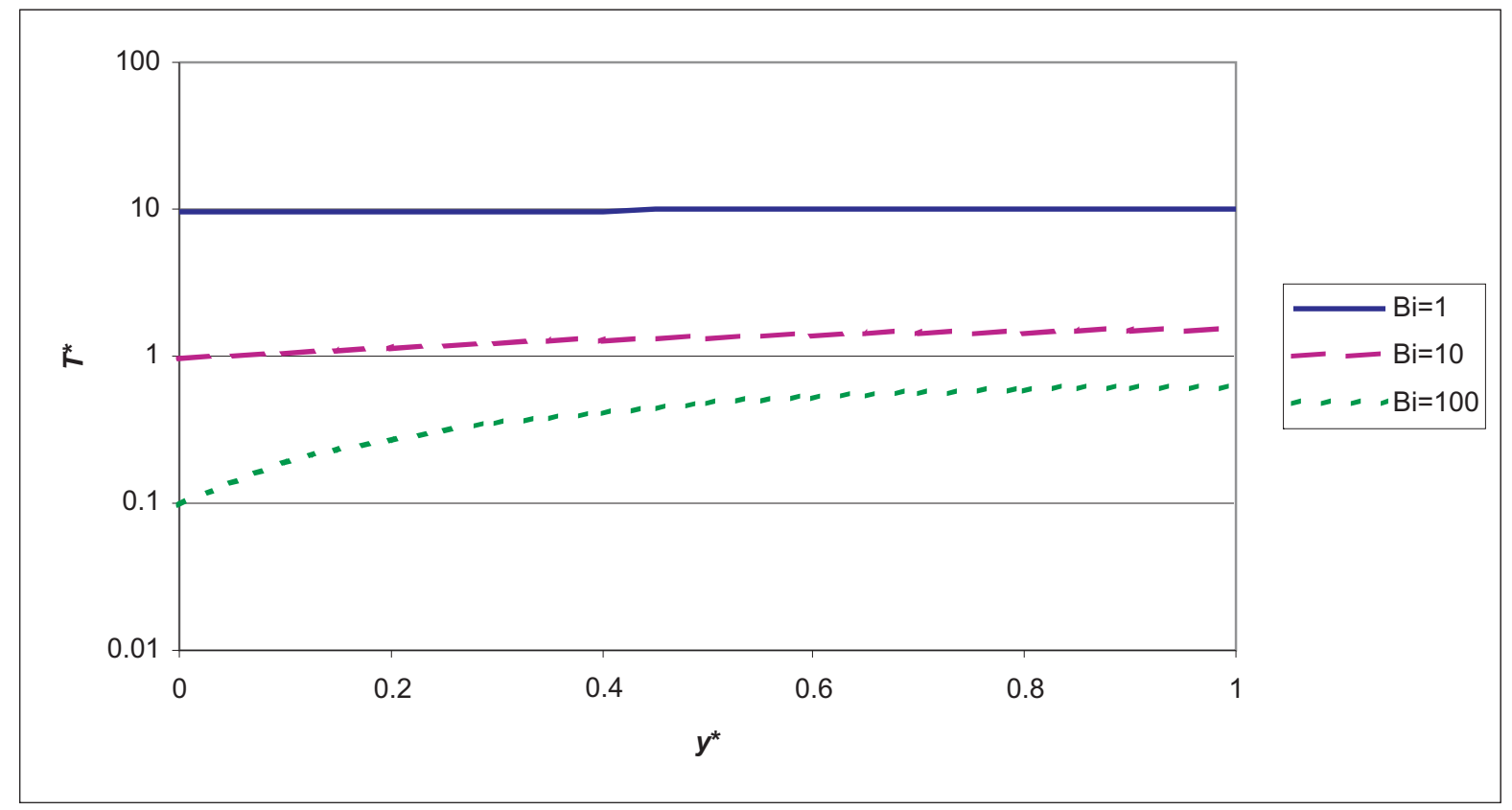

Fig. 2: Dimensionless temperature profiles

$$
T-T_{\mathrm{f}}=\frac{\mu_{\mathrm{p}} H^{2} \dot{\gamma}^{2}}{\lambda}\left(1+\tau_{0}^{*}\right)\left(\frac{y}{H}-\frac{1}{2}\left(\frac{y}{H}\right)^{2}+\frac{1}{B i}\right),
$$

The solution of (9) for $B i \rightarrow \infty$ is shown in graphical form in Fig. 3, where

$$
T^{*}=\frac{\left(T-T_{\mathrm{f}}\right) \lambda}{\mu_{\mathrm{p}} H^{2} \dot{\gamma}^{2}} .
$$

where

$$
\tau_{0}^{*}=\frac{\tau_{0}}{\mu_{\mathrm{p}} \dot{\gamma}} .
$$

Fig. 3 shows that increasing plasticity $\left(\tau_{0}^{*}\right)$ increases the temperature rise.

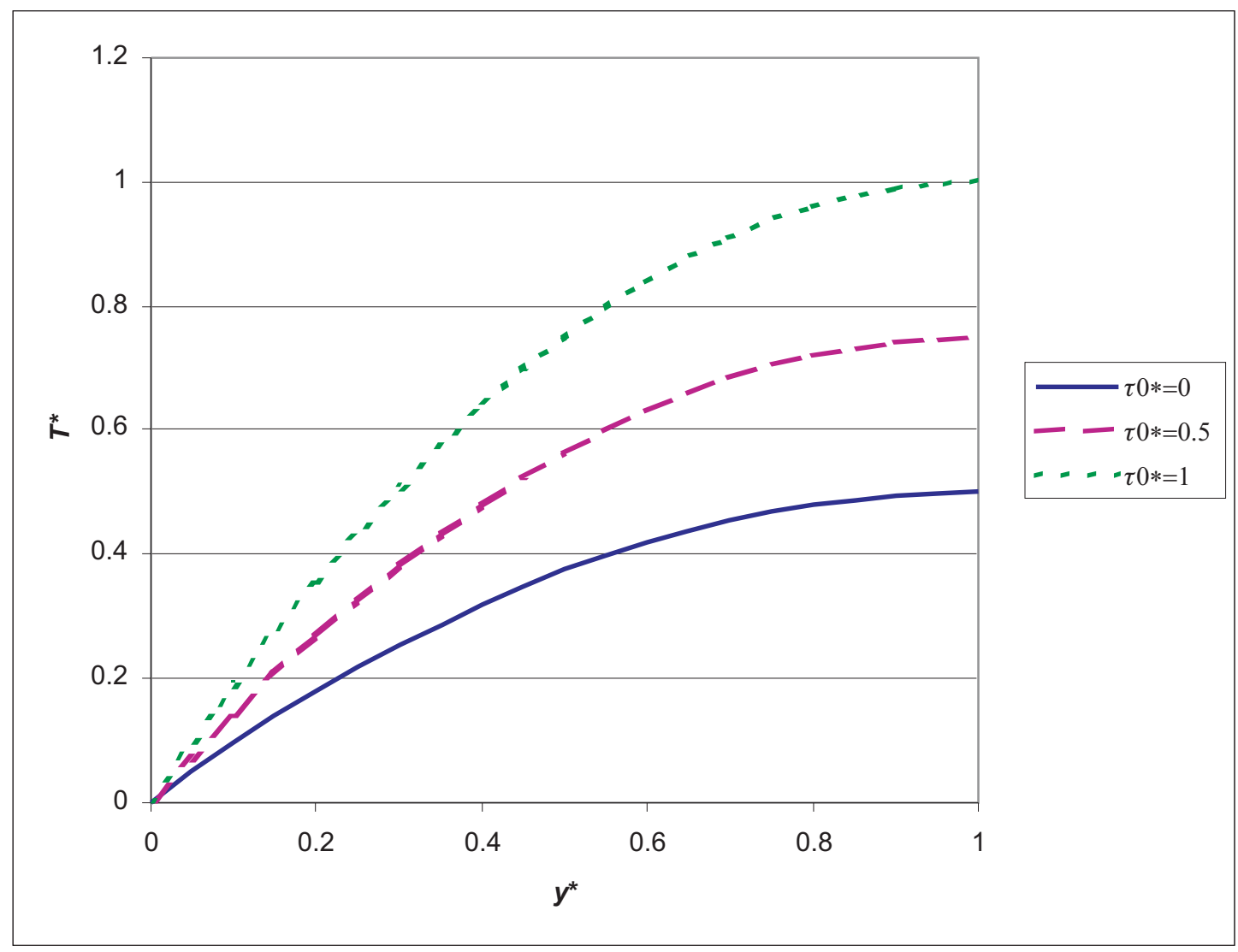

Fig. 3: Dimensionless temperature profiles 


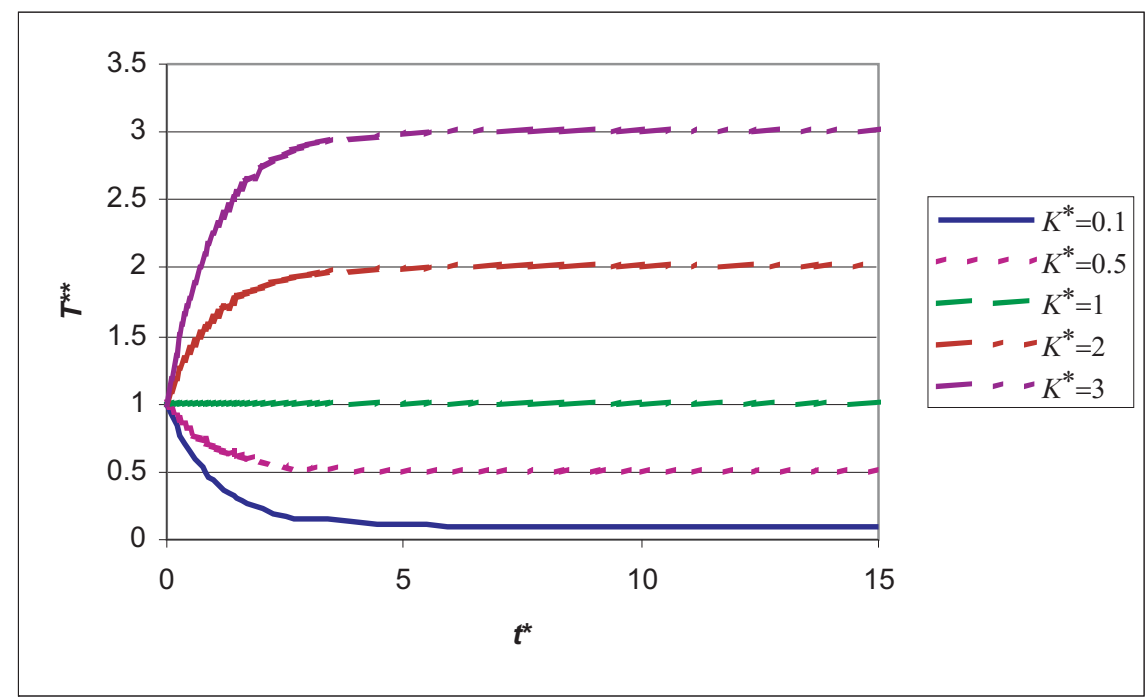

Fig. 4: Dependence of dimensionless temperature on dimensionless time

From the dependencies shown in Fig. 2 it can be seen that at small Biot number values the outer temperature resistance prevails and the temperature of the liquid is practically constant, and in a unsteady-state it depends on time. For this case the enthalpy balance can be written in the form

$$
\rho c H \frac{\mathrm{d} T}{\mathrm{~d} t}=\tau \dot{\gamma} H-\alpha\left(T-T_{\mathrm{f}}\right)
$$

and after integration it transforms to

$T^{* *}=\frac{T-T_{\mathrm{f}}}{T_{0}-T_{\mathrm{f}}}=K^{*}+\left(1-K^{*}\right) \exp \left(-t^{*}\right)$,

where

$$
K^{*}=\frac{t \dot{\gamma} H}{\alpha\left(T_{0}-T_{\mathrm{f}}\right)}, \quad t^{*}=\frac{\alpha t}{\rho c H} .
$$

The dependence is shown in Fig. 4, where the line for $K^{*}=1$ designates the equilibrium state at which all dissipative heat is removed by convection.

The dependence of the dimensionless time $t^{*}$ after which the dimensionless temperature attains $99 \%$ of the steady-state value on $K^{*}$ is shown in Fig. 5.
In the case when initial temperature $T_{0}$ is equal to temperature $T_{\mathrm{f}}$ it is suitable to define the dimensionless temperature as

$$
T^{+}=\frac{\left(T-T_{\mathrm{f}}\right) \alpha}{t \dot{\gamma} H}
$$

after integration (11) we can obtain the relation

$$
T^{+}=1-\exp \left(-t^{*}\right) .
$$

The application of the above relations will be illustrated in the following example.

\section{Example}

A rotational viscometer with inner cylinder diameter $48 \mathrm{~mm}$ and outer cylinder diameter $50 \mathrm{~mm}$ contains a Newtonian liquid with density $\rho=1000 \mathrm{kgm}^{-3}$, heat capacity $c=4200 \mathrm{Jkg}^{-1} \mathrm{~K}^{-1}$ and heat conductivity $\lambda=0.5 \mathrm{Wm}^{-1} \mathrm{~K}^{-1}$. Calculate the inner and outer cylinder temperature

1) when the tempering temperature is $20^{\circ} \mathrm{C}$ and the heat transfer coefficient $\alpha=100 \mathrm{Wm}^{-2} \mathrm{~K}^{-1}$

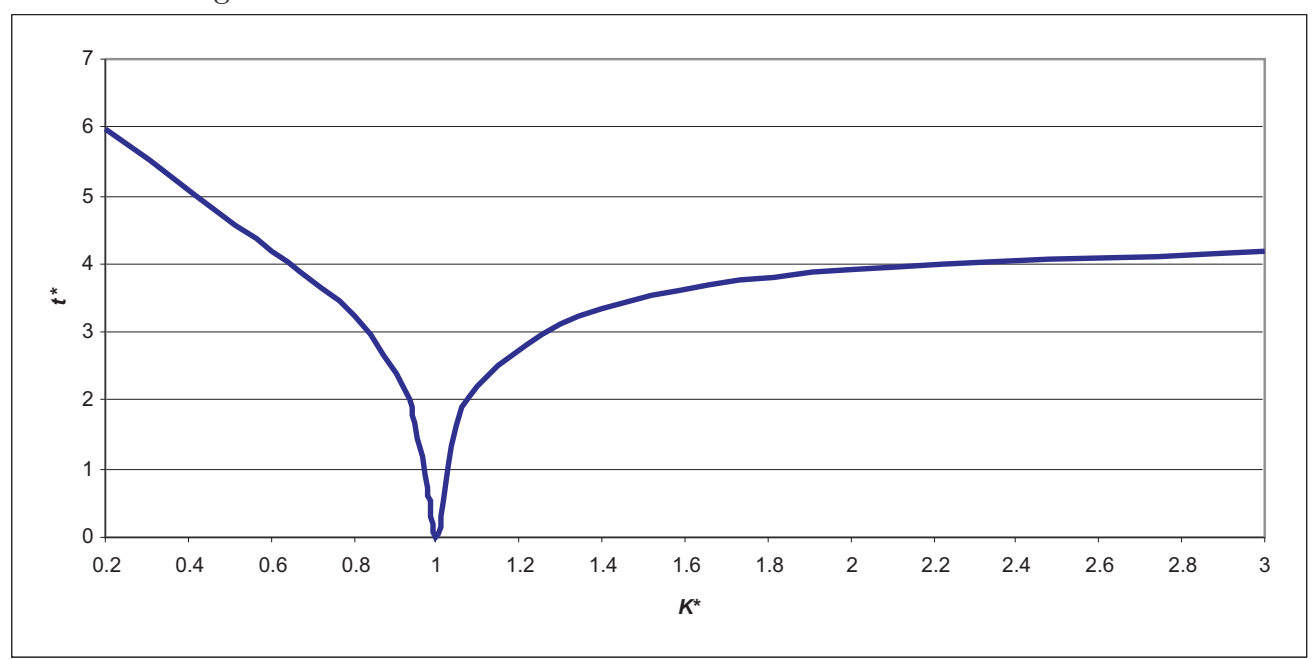

Fig. 5: Dependence of the dimensionless time $t^{*}$ after which the dimensionless temperature attains $99 \%$ of the steady-state value on $K^{*}$ 
2) at no tempering, outer temperature $20^{\circ} \mathrm{C}$ and $\alpha=5 \mathrm{Wm}^{-2} \mathrm{~K}^{-1}$. The temperature dependence of viscosity is described by the relation

$$
\mu[\mathrm{Pa} \cdot \mathrm{s}]=0.82 \exp \left(-0.025 T\left[{ }^{\circ} \mathrm{C}\right]\right) .
$$

Measurement is carried out at shear rate
a) $100 \mathrm{~s}^{-1}$,
b) $1000 \mathrm{~s}^{-1}$.

Calculate also the temperature after 5 minutes, when the initial temperature is
a) $20^{\circ} \mathrm{C}$,
B) $15{ }^{\circ} \mathrm{C}$.

\section{Solution}

\subsection{Calculations of final temperatures}

First the Biot number will be calculated

1) $B i=\frac{\alpha H}{\lambda}=\frac{100 \times 0.001}{0.5}=0.2$.

Inserting $n=1$ and $K=\mu$ into Eq. (6) or inserting $\tau_{0}^{*}=0$ and $\mu_{\mathrm{p}}=\mu$ into Eq. (9), the inner cylinder temperature $T_{\mathrm{i}}$ will be calculated for $y=H$ from

a)

$$
\begin{aligned}
T_{\mathrm{i}} & =\frac{\mu H^{2} \dot{\gamma}^{2}}{\lambda}\left(\frac{1}{2}+\frac{1}{B i}\right)+T_{\mathrm{f}} \\
& =\frac{0.5 \times 10^{-6} \times 10^{4}}{0.5} 5.5+20=20.055^{\circ} \mathrm{C}
\end{aligned}
$$

and the outer cylinder temperature $T_{\mathrm{e}}$ will be calculated for $y=0$ from

$$
\begin{aligned}
T_{\mathrm{e}} & =\frac{\mu H^{2} \dot{\gamma}^{2}}{\lambda}\left(0+\frac{1}{B i}\right)+T_{\mathrm{f}} \\
& =\frac{0.5 \times 10^{-6} \times 10^{4}}{0.5} 5+20=20.05^{\circ} \mathrm{C} .
\end{aligned}
$$

b)

$$
\begin{aligned}
T_{\mathrm{i}} & =\frac{\mu H^{2} \dot{\gamma}^{2}}{\lambda}\left(\frac{1}{2}+\frac{1}{B i}\right)+T_{\mathrm{f}} \\
& =\frac{0.44 \times 10^{-6} \times 10^{6}}{0.5} 5.5+20=24.9^{\circ} \mathrm{C}, \\
T_{\mathrm{e}} & =\frac{\mu H^{2} \dot{\gamma}^{2}}{\lambda} \frac{1}{B i}+T_{\mathrm{f}} \\
& =\frac{0.44 \times 10^{-6} \times 10^{6}}{0.5} 5+20=24.4^{\circ} \mathrm{C} .
\end{aligned}
$$

The viscosities at the mean liquid temperature were inserted into the above equations.

2) $B i=\frac{\alpha H}{\lambda}=\frac{5 \times 0.001}{0.5}=0.01$

and the two temperatures will be calculated from the same equations

a)

$$
\begin{aligned}
T_{\mathrm{i}} & =\frac{\mu H^{2} \dot{\gamma}^{2}}{\lambda}\left(\frac{1}{2}+\frac{1}{B i}\right)+T_{\mathrm{f}} \\
& =\frac{0.485 \times 10^{-6} \times 10^{4}}{0.5} 100.5+20=21^{\circ} \mathrm{C},
\end{aligned}
$$

$$
\begin{aligned}
T_{\mathrm{e}} & =\frac{\mu H^{2} \dot{\gamma}^{2}}{\lambda}\left(0+\frac{1}{B i}\right)+T_{\mathrm{f}} \\
& =\frac{0.485 \times 10^{-6} \times 10^{4}}{0.5} 100+20=21^{\circ} \mathrm{C} .
\end{aligned}
$$

b)

$$
\begin{aligned}
T_{\mathrm{i}} & =\frac{\mu H^{2} \dot{\gamma}^{2}}{\lambda}\left(\frac{1}{2}+\frac{1}{B i}\right)+T_{\mathrm{f}} \\
& =\frac{0.19 \times 10^{-6} \times 10^{6}}{0.5} 100.5+20=58.3^{\circ} \mathrm{C}, \\
T_{\mathrm{e}} & =\frac{\mu H^{2} \dot{\gamma}^{2}}{\lambda} \frac{1}{B i}+T_{\mathrm{f}} \\
& =\frac{0.19 \times 10^{-6} \times 10^{6}}{0.5} 100+20=58.2^{\circ} \mathrm{C} .
\end{aligned}
$$

These results show that at a high shear rate the temperature rise is unacceptable especially without tempering. It can also be seen that the difference between the inner and outer cylinder temperature is not high due to the low Biot number values, especially in case 2 ).

\subsection{Calculations of temperatures after 5 minutes of measurement}

$\alpha)$ In the case when the initial temperature $T_{0}$ is equal to temperature $T_{\mathrm{f}}$, Eq.(15) will be usedin the calculations

1a)

$$
\begin{aligned}
T & =\frac{\mu H \dot{\gamma}^{2}}{\alpha}\left[1-\exp \left(-t^{*}\right)\right]+T_{\mathrm{f}} \\
& =\frac{0.497 \times 0.001 \times 100^{2}}{100}\left[1-\exp \frac{-100 \times 300}{1000 \times 4200 \times 0.001}\right]+20 \\
& =20.05^{\circ} \mathrm{C}
\end{aligned}
$$

1b)

$$
\begin{aligned}
T & =\frac{\mu H \dot{\gamma}^{2}}{\alpha}\left[1-\exp \left(-t^{*}\right)\right]+T_{\mathrm{f}} \\
& =\frac{0.47 \times 0.001 \times 1000^{2}}{100}\left[1-\exp \frac{-100 \times 300}{1000 \times 4200 \times 0.001}\right]+20 \\
& =24.7^{\circ} \mathrm{C},
\end{aligned}
$$

2a)

$$
\begin{aligned}
T & =\frac{\mu H \dot{\gamma}^{2}}{\alpha}\left[1-\exp \left(-t^{*}\right)\right]+T_{\mathrm{f}} \\
& =\frac{0.5 \times 0.001 \times 100^{2}}{5}\left[1-\exp \frac{-5 \times 300}{1000 \times 4200 \times 0.001}\right]+20
\end{aligned}
$$$$
=20.3^{\circ} \mathrm{C} \text {, }
$$

2b)

$$
\begin{aligned}
T & =\frac{\mu H \dot{\gamma}^{2}}{\alpha}\left[1-\exp \left(-t^{*}\right)\right]+T_{\mathrm{f}} \\
& =\frac{0.375 \times 0.001 \times 1000^{2}}{5}\left[1-\exp \frac{-5 \times 300}{1000 \times 4200 \times 0.001}\right]+20 \\
& =42.5^{\circ} \mathrm{C},
\end{aligned}
$$

where viscosity was calculated at the mean temperature $\left(T+T_{\mathrm{f}}\right) / 2$. 
$\beta$ ) In cases when the initial temperature $T_{0}$ is not equal to temperature $T_{\mathrm{f}}$, Eq.(12) will be used in the calculations

1a)

$$
\begin{aligned}
T & =T_{\mathrm{f}}+\left(T_{0}-T_{\mathrm{f}}\right)\left[K^{*}+\left(1-K^{*}\right) \exp \left(-t^{*}\right)\right] \\
& =20-5\left[(1+0.0106) \exp \left(-t^{*}\right)-0.0106\right]=20.05^{\circ} \mathrm{C},
\end{aligned}
$$

1b)

$$
\begin{aligned}
T & =T_{\mathrm{f}}+\left(T_{0}-T_{\mathrm{f}}\right)\left[K^{*}+\left(1-K^{*}\right) \exp \left(-t^{*}\right)\right] \\
& =20-5\left[(1+0.995) \exp \left(-t^{*}\right)-0.995\right]=25^{\circ} \mathrm{C},
\end{aligned}
$$

2a)

$$
\begin{aligned}
T & =T_{\mathrm{f}}+\left(T_{0}-T_{\mathrm{f}}\right)\left[K^{*}+\left(1-K^{*}\right) \exp \left(-t^{*}\right)\right] \\
& =20-5\left[(1+0.22) \exp \left(-t^{*}\right)-0.22\right]=16.8^{\circ} \mathrm{C},
\end{aligned}
$$

2b)

$$
\begin{aligned}
T & =T_{\mathrm{f}}+\left(T_{0}-T_{\mathrm{f}}\right)\left[K^{*}+\left(1-K^{*}\right) \exp \left(-t^{*}\right)\right] \\
& =20-5\left[(1+16.3) \exp \left(-t^{*}\right)-16.3\right]=41^{\circ} \mathrm{C},
\end{aligned}
$$

where viscosity was calculated at the mean temperature $\left(T_{0}+T\right) / 2$

The results presented above show that the temperature rise and the experimental error is considerable especially at high shear rate in cases $1 b$ ) and $2 b$ ).

\section{Conclusion}

It was shown that dissipative heating can play an important role in measurement of highly viscous fluids. The temperature of measured liquid can be significantly higher than tempering temperature, which can cause significant experimental error. The time necessary for temperature stabilisation is often not negligible. Measurement without tempering can lead to a significant temperature rise and unacceptable error of measurement.

\section{List of symbols}

Bi Biot number, 1

c specific heat capacity, $\mathrm{J} \cdot \mathrm{kg}^{-1} \cdot \mathrm{K}^{-1}$

$H \quad$ distance of planes (cylinder walls), $\mathrm{m}$

$K \quad$ consistency coefficient, $\mathrm{Pa} \cdot \mathrm{s}^{\mathrm{n}}$

$n$ flow index, 1
$R_{1} \quad$ inner cylinder radius, $\mathrm{m}$

$R_{2} \quad$ outer cylinder radius, $m$

$t$ time, s

$T$ temperature, $\mathrm{K}$

y coordinate, $\mathrm{m}$

$\alpha \quad$ heat transfer coefficient, $\mathrm{W} \cdot \mathrm{m}^{-2} \cdot \mathrm{K}^{-1}$

$\dot{\gamma} \quad$ shear rate, $\mathrm{s}^{-1}$

$\kappa \quad$ ratio $\mathrm{R} 1 / \mathrm{R} 2,1$

$\lambda$ heat conductivity, $\mathrm{W} \cdot \mathrm{m}^{-1} \cdot \mathrm{K}^{-1}$

$\mu \quad$ viscosity, Pa.s

$\mu_{\mathrm{p}} \quad$ plastic viscosity, $\mathrm{Pa} \cdot \mathrm{s}$

$\rho \quad$ density, $\mathrm{kg} \cdot \mathrm{m}^{-3}$

$\tau \quad$ shear stress, $\mathrm{Pa}$

$\tau_{0} \quad$ yield stress, $\mathrm{Pa}$

lower indexes

f fluid

0 initial

upper indices

* dimensionless

\section{Acknowledgment}

This work was supported by research project of the Ministry of Education of the Czech Republic MSM6840770035.

\section{Reference}

[1] Bird, R. B., et al.: Transport Phenomena, J. Wiley, N. York, 1960.

Prof. Ing. František Rieger, DrSc.

Phone: +420 224352548

e-mail: frantisek.rieger@fs.cvut.cz

Department of Process Engineering

Czech Technical University in Prague

Faculty of Mechanical Engineering

Technická 4

16607 Prague 6, Czech Republic 